\title{
Role of Curcuma longa in the Management of Gingivitis
}

\author{
Umesh Pratap Verma, Gupta Abhaya, Sharma Disha \\ Department of Periodontology, Faculty of Dental Sciences, King George's Medical University, India
}

\begin{tabular}{l}
\hline \hline Article Info \\
\hline Article history: \\
ReceivedAug 2, 2018 \\
Revised Sep 6, 2018 \\
Accepted Sep 15, 2018 \\
\hline
\end{tabular}

Keyword:

Anti-inflammatory

Bleeding on probing

Curcuma longa

Gingival score

Gingivitis

Probing depth

\begin{abstract}
The most prevalent form of periodontal disease is gingivitis. The bleeding on probing, erythema, edema and ulceration are important signs of gingivitis. Previously conventional periodontal therapy has relied almost exclusively upon mechanical debridement of tooth surface. But as of today conventional periodontal therapy alone is not sufficient because pathogenic bacteria has been demonstrated not only in gingival tissue but even up to the crest of alveolar bone itself. Because of the side effect of non-steroidal antiinflammatory drug, the use of medicinal plants for treatment of this disease was advocated. Amongst the herbs, Curcuma longa belonges to family Zingiberaceae, commonly available in India and used in various domestic affairs popular as Haldi. The present study has been conducted with aims to evaluate the anti-inflammatory effect of extract of Curcuma longa as topical and irrigant in various concentrations $(0.5 \& 1 \%)$ on inflamed gingival tissue of patient with gingivitis. All the subjects were divided into two groups. Group one was control, Group two (Test Group) was further subdivided in to IIa and IIb. Results revealed the extract of Curcuma longa play a significant role in reducing the inflammatory response of the gingiva like reduced gingival score, bleeding on probing and probing depth.
\end{abstract}

Copyright $@ 2018$ Institute of Advanced Engineering and Science. All rights reserved.

\section{Corresponding Author:}

Umesh PratapVerma,

Department of Periodontology, Faculty of Dental Sciences,

King George's Medical University,

Lucknow, UP, India.

Email: umeshpratapverma@kgmcindia.edu

\section{INTRODUCTION}

Herbal medicine is the use of medicinal plants for treatment and prevention of diseases: it ranges from traditional and accepted medicines of every country to utilize standardized and titrated herbal extracts [1]. Medicinal plants are widely used as home remedies and raw materials for the pharmaceutical industries. Herbal remedies are used in the prevention, treatment and cure of disorders and diseases since ancient times. However, use of medicinal herbs may not meet the requirements of quality, safety and efficacy [2]. There are plenty of medicinal plants which have been helpful like Neem bark radical scavenging, carcinogen-detoxification, anti-inflammatory, anti-angiogenic [3], Amla used as antioxidant [4], Chirita used as digestive, astringent [5], Guggul is used as obesity and hyperlipidemia [6]. The twig of Acacia arabica known as Babool is used for cleansing of teeth, which has antimicrobial and antiplaque activities [7].

Curcumin is a natural polyphenol and essential curcuminoid derived from the rhizome of the medicinal plant Curcuma longa (L.) is universally acknowledged as "Wonder drug of life" [8]. Curcuma longa is commonly found herbs in India used in household purposes and is commonly called as 'haldi' with Zingiberaceae family. It is mostly used as a spice, a colouring agent and broadly used in traditional medicine such as Ayurveda, Unani, etc [9]. It is used as hypolipidemic, anti-inflammatory, anti-diarrhoeal, hepatoprotective, anti-asthmatic and anti-cancerous drug, cosmetology etc. [10]. Curcumin, the most 
biologically active curcuminoid in turmeric, is being investigated in pre-clinical and clinical trials for its role in disease prevention and cure [11].

Gingivitis affects an approximate $80 \%$ of the population and is considered as the world's most predominant inflammatory periodontal disease. Without interference, gingivitis can advance to alveolar bone loss. Thus, the primary objective in patients with gingivitis is to control plaque buildup and inflammation of soft tissue. Turmeric, a commonly used herb contains anti-inflammatory, antioxidant, antibacterial, antiviral, and antifungal properties. By benefit of these properties, multiple controlled trials were completed to investigate the efficacy of turmeric in treating gingivitis $[12,13]$. Turmeric and curcumin both are nontoxic for human especially the oral administration. Turmeric and curcumin are also found safe in animals. Both are non mutagenic and are safe in pregnancy in animals but more studies in human are required [14]. Gingivitis is condition caused by substances derived from microbial plaque compiling at or near gingival sulcus; all other assumed local and systemic etiologic factors either augment plaque retention, or enhance the susceptibility of gingival tissue to microbial attack.

Some of the basic symptoms of gingivitis are bleeding gums that are reddened or swollen are the major signs of gingivitis. The gums mainly bleed for the period of tooth brushing, but they may also bleed spontaneously. Healthy gums are generally coral pink, while inflamed gums take on a red to dark-red colour. Chronic Gingivitis generally causes no pain or any other major symptom and is undetected for some time but it may spread to other areas of periodontium and cause harm there. That is referred to as periodontitis. Over time it can untie teeth, and it may become impossible to protect them [15]. The research aimed to explore the clinical efficacy of different concentrations of Curcumin $(0.5 \% \& 1 \%)$ on inflamed gingiva of moderate to severe gingivitis patients.

\section{RESEARCH METHOD}

Fresh rhizomes were cleaned and washing was done with deionised water, sliced and dehydrated in the sun for one week and then again dried at $50^{\circ} \mathrm{C}$ in a hot air oven for about 6 hours and were sliced into small pieces with help electronic mill. Approximately $20 \mathrm{gm}$ of sample were taken into a thimble and placed in a soxhlet apparatus, were set up with various solvent from non- polar to polar. $150 \mathrm{ml}$ of solvent was added and extracted according to their boiling point for 6 hours. The solvents used were Hexane (B.P=69 ${ }^{\circ}$ C), Chloroform $\left(B \cdot P=61^{\circ} \mathrm{C}\right)$, Ethyl acetate $\left(B \cdot P=77^{\circ} \mathrm{C}\right)$, Methanol $\left(B \cdot P=65^{\circ} \mathrm{C}\right)$, and Acetone $\left(B \cdot P=56 \cdot 53^{\circ} \mathrm{C}\right)$. One sample was extracted with hexane for 2 hours and hexane extract was discarded and the powder was reextracted with methanol for 6 hours.After finishing the extraction process, dark brown extract was cooled, concentrated, filtered by means of the rotary evaporator, and finally by vacuum suction to get the crude dried out extract which was like black orange in colour. Each raw segment of turmeric was isolated by the same process and result was calculated. The individual Curcuminoids collected from the column chromatography was dissolved in the methanol solution and heated. After entire dissolution added chloroform to get ratio of methanol: chloroform 5:2 and kept at $5^{\circ} \mathrm{C}$ for overnight. The crystals obtained were detached by filtration [16].

The study was conducted in 30 subjects (male+female) in the age group of 25 to 45 years from the Outpatient department of Periodontitis, Faculty of Dental Sciences, King George's Medical University (KGMU), Lucknow, with collaboration of National Botanical Research Institute (NBRI), Lucknow. Institutional ethical clearance was issued from ethical committee of King George's Medical University (KGMU), Lucknow.

Verbal and written informed consent was obtained from every participant. This pilot study comprises of randomly selected participants by inclusion and exclusion selection criterias. The inclusion criterias included: 1) Systematically healthy subjects; 2) Moderate to severe gingivitis [17]; 3) Facial surface of maxillary and mandibular anterior teeth; 4)No periodontal therapy for last six months. The exclusion criteria included: 1) Acute oral infection, carious teeth, malocclusion and wasting disease; 2) Betel, tobaccochewing habits; 3) On any drug therapy for the last six months; 4) Lactating and Pregnant women.

All the subjects were divided in to two groups through randomization (computer assisted). Then Group I i.e. control (SRP-scaling and root planing) and Group II i.e. experimental. Group II was further subdivided into two sub groups, Group II a (SRP+Topical\&Gingival sulcular irrigation by $0.5 \%$ Curcumin); $\mathrm{n}=10$ and Group II b (SRP+Topical\& Gingival sulcular irrigation by $1 \%$ Curcumin); $\mathrm{n}=10$. SRP was performed by ultrasonic scalar and meticulous root planning by gracey curettes until glossy hard surface was obtained.In Group IIa\&IIb topical application was done by cotton pellet soaked in respective curcumin solutions (i.e Group IIa with $0.5 \%$ and Group IIb with $1 \%$ Curcumin solution) along with gingival sulcular irrigation with the same solution. The application procedure was repeated daily for 6 consecutive days. All the subjects were also instructed to leave the solution in the mouth for at least $10 \mathrm{~min}$ after application and thereafter rinsed with water to clear any residual medication. Further, Gingival Index and Gingival bleeding 
index were recorded at Baseline, 7th, 14th, 21st and 28th using mouth mirror, explorer, UNC- 15 probe and tweezer.

Several parameters were taken into consideration for assessment of gingivitis, bleeding on probing. These clinical parameters were recorded at $0,7,14,21$ and 28 :

a. Change in Mean Gingival Score

b. Change in Mean Bleeding on Probing

Gingival Score: Loe and Silness (1963) method for assessment of gingivitis was considered:

$0=$ Normal gingiva

$1=$ mild inflammation, slight change, oedema, no bleeding on palpation

$2=$ moderate inflammation, redness, oedema, and glazing, bleeding on probing

$3=$ severe inflammation, redness, oedema, ulceration, tendency of spontaneous bleeding.

Ainamo and Bay (1975) method [18] of bleeding on probing was considered. The presence and absence of gingival bleeding was determined by gentle probing of the gingival crevice with a periodontal probe. The appearance of bleeding within $10 \mathrm{sec}$ was indicated as positive score $(+(1))$ and no bleeding as negative score (-(0)).

The statistical evaluation was performed using Mean, Median, Standard deviation and student t-test appropriately. The software used for all statistical evaluation was SPSS 17 statistical package program. Test of significance $(p)$ is the difference between the mean were evaluated for their significance. Results were considered to be significantly difference for any ' $\mathrm{p}$ ' value $<0.005$.

\section{RESULT}

All 30 patients were completed the entire follow up period of the study. The experimental solution had a good patient's acceptance and did not show adverse reactions such as ulceration or allergic reactions. At baseline, all individuals had significantly higher bleeding on probing and gingival score. On day 7 there was reduction in the bleeding on probing and gingival score as well.

Group 1 (Control), Figure 1 (a) showed the gross calculus and plaque deposition. Upper incisors were protruded and the edematous generalized gingival swellings with drifting of teeth were observed. Post treatment as shown in Figure 1 (b) with scaling and root planning showed uneventful healing after 28 days. Group 2 (a) as shown in Figure 2, pre-operative view displayed gross calculus, plaque, generalized gingival redness and some spontaneous bleeding areas in the entire lower and upper arch. After scaling and root planning with local and sub gingival irrigation of $0.5 \%$ curcumin showed excellent healing and achieved normal colour and contour of gingiva after 28 days. Group 2 (b) as shown in Figure 3, at base line clinical picture showed generalized gingival overgrowth with redness and friable gingival texture which was bleeding spontaneously or slight provocation. Highly appreciable post-operative 28 days shown in Figure 3 (b) results were obtained by using $1 \%$ curcumin as local and sub gingival irritant using 16 gauze needle with scaling and root planning.

Figure 4 on day 7 there was reduction in the bleeding on probing in group I, group IIa and group IIb. On day 14 there was significant reduction in bleeding on probing in group IIa and group IIb as compared to group I which increased significantly from days 21 and 28 . The mean change in the bleeding on probing was with group I was $0.34 \pm 0.8$ with group IIa $0.41 \pm 0.9$ with group IIb $0.54 \pm 0.75$. Figure 5 on day 7 there was reduction in the Gingival Score in group I, group IIa and group IIb. On day 14 there was significant reduction in Mean Gingival Score when compared group Iwith group IIa and group IIb which displayed similar trend from 14, 21 and 28 days. The mean change in the Mean Gingival Score in group I was $0.35 \pm 0.12$, group IIa $0.45 \pm 0.9$ and with group IIb $0.50 \pm 0.8$.

The confidence limit of patients was good. Clinically there was significant change in the colour of the gingiva from reddish to coral pink. The readings are tabulated considering the pre interventional and post interventional scores for papillary bleeding index. The mean, standard deviation and test of significance were studied using Student " $\mathrm{t}$ " test and Test of significance (p). Results were considered to be statistically significant for any 'p' value $<0.005$. All the individuals in this current study exhibited appreciable antigingivitis activity determined from the clinical parametric analysis. 


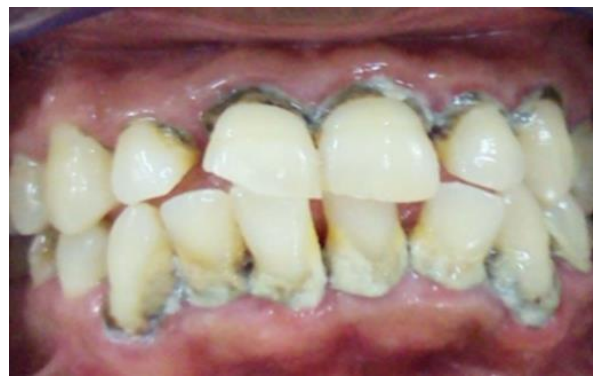

(a)

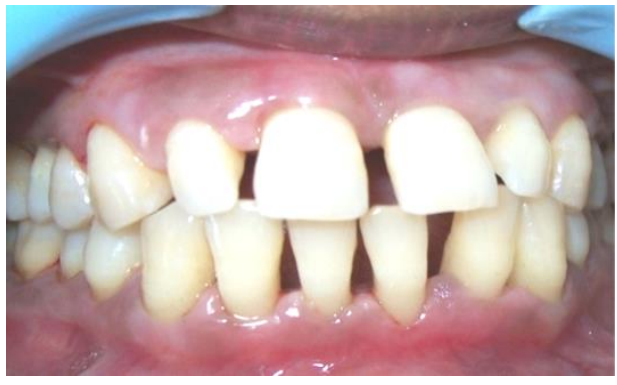

(b)

Figure 1. (a) Showed the gross calculus and plaque deposition. Upper incisors were protruded and the edematous generalized gingival swellings with drifting of teeth were observed. (b) Post treatment with scaling and root planning showed uneventful healing after 28 days

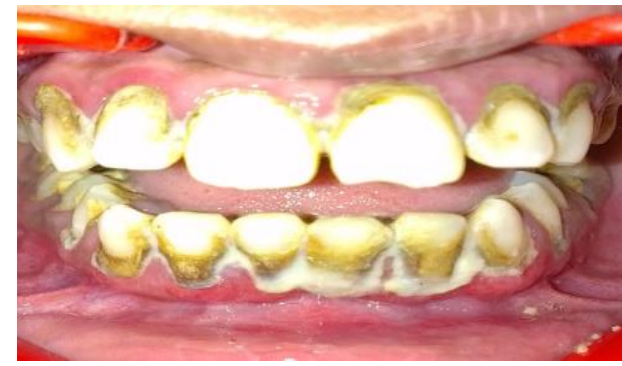

(a)

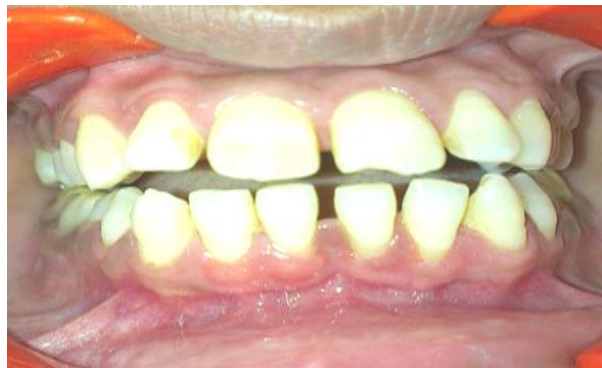

(b)

Figure 2. (a) Pre-operative view displayed gross calculus, plaque, generalized gingival redness and some spontaneous bleeding areas in the entire lower and upper arch. (b) After scaling and root planning with local and sub gingival irrigation of $0.5 \%$ curcumin showed excellent healing and achieved normal colour and contour of gingiva after 28 days

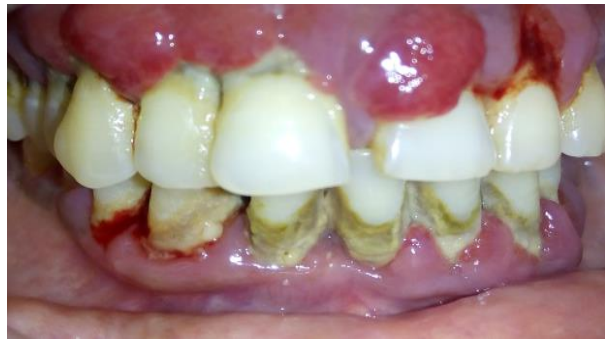

(a)

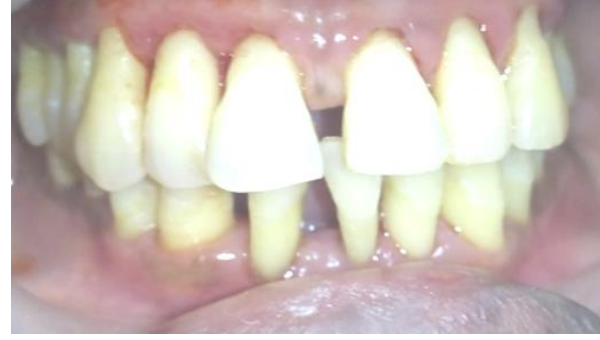

(b)

Figure 3. (a) At base line clinical picture showed generalized gingival overgrowth with redness and friable gingival texture which was bleeding spontaneously or slight provocation. (b) Highly appreciable postoperative 28 days results were obtained by using $1 \%$ curcumin as local and sub gingival irritant using 16 gauze needle with scaling and root planning. 


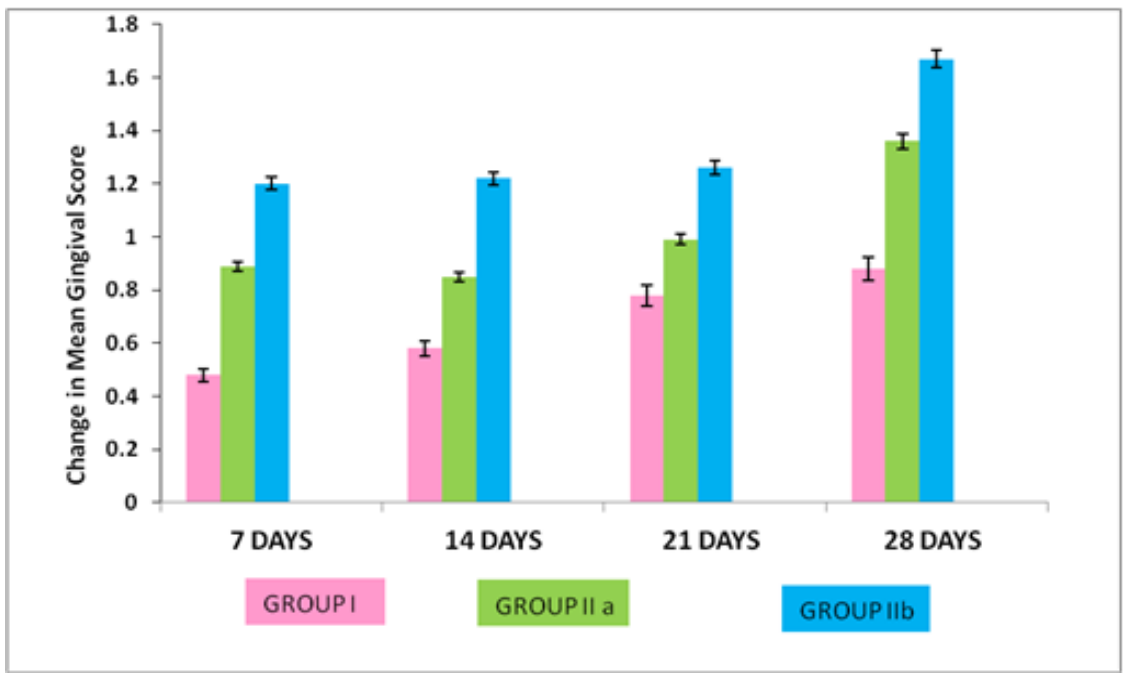

Figure 4. Change in Mean Bleeding on Probing in different groupsin different periods

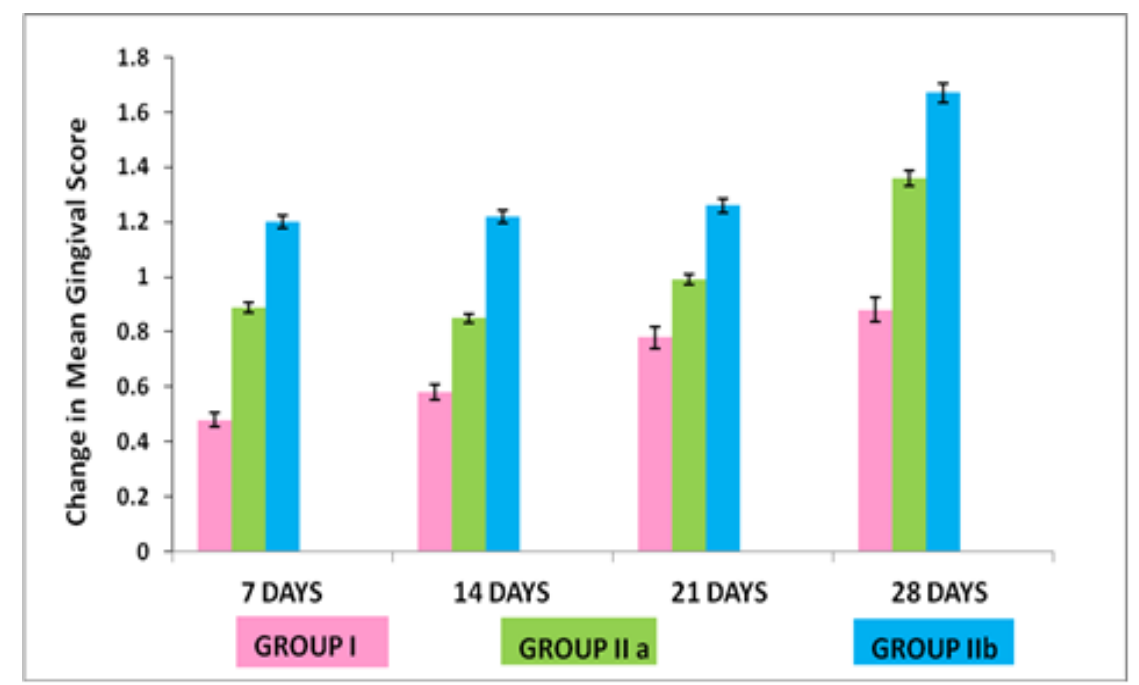

Figure 5. Change in Mean Gingival Score in different groups

\section{DISCUSSION}

The reason for using the herbal product curcumin is based on the fact that gingival disease is result of interaction between bacteria and its product which results in inflammatory and immunological changes within the periodontal tissues. There are various chemicals which can be highlighted by various researchers, but they have its side effects then benefits. Curcuminoids and other chemicals constituents of turmeric are well known for anti-inflammatory activity [19]. In our study we have noted that the inflammation has been reduced from 7th to 28th day continuously. It can be due to free radical scavenging antioxidant property [20]. In recent research, it was stated that curcumin inhibited 97.3\% lipid peroxidation of linoleic acid emulsion at $15 \mathrm{microg} / \mathrm{mL}$ concentration $(20 \mathrm{mM})$. In count, curcumin was found effective in DPPH (1,1- diphenyl-2picryl- hydrazyl free radical) scavenging, in DMPD (N,N-dimethyl-p-phenylene diamine dihydrochloride ) scavenging, superoxide anion radical scavenging, hydrogen peroxide scavenging, ABTS (2,2'-azino-bis(3ethylbenzthiazoline-6-sulfonic acid) scavenging, ferric ions $(\mathrm{Fe}(3+))$ reducing power by the transformation method and ferrous ions $(\mathrm{Fe}(2+))$ which is an chelating actions. In addition, BHA (butylated hydroxyanisole), BHT (butylated hydroxytoluene), alpha-tocopherol and trolox, can be used as the reference to an antioxidant and radical scavenging compounds. This depicted that, curcumin can be used in the pharmacological and food industry as it has these properties [20]. Curcumin is a pharmacologically secure compound with anticarcinogenic, anti-inflammatory and possess free radical scavenging properties. In this study, we examined 
the positive alterations in gingival score that might be the effects of curcumin on the actions of various phospholipases that occupied in different signalling pathways. The anti-inflammatory effects of curcumin on enzyme activities of these phospholipases in a cell-free system: G protein-mediated phospholipase D (PLD), phosphatidylinositol-specific phospholipase C, and phospholipase A. Curcumin inhibited various types of phospholipases, most efficiently PLD among those experienced. It also inhibited 12-O-tetradecanoylphorbol13-acetate-induced PLD foundation as dose-dependent manner in animals. These results suggest that the antiinflammatory and anti-carcinogenic action of curcumin is partly due to the inhibition of PLD [21].

Different concentration $(0.5,1.0 \%)$ of curcuma longa was used to see the optimum concentration with maximum efficiency for the periodontal patients. Which was applied for 6 days regularly and its effect was seen in weekly interval till end of 4 th week (28 days). In recent research the oral formulation containing C. longa extract is effective in treating early infective-inflammatory periodontal diseases not only when used as an adjunct to SRP (scaling and root planing) but also when used alone. Mean significant reduction was observed in PI, GI, PD and increased in clinical attachment level were demonstrated in mutually the groups from baseline to 45 days. Curcumin can be efficiently used along with scaling and root planing [22]. On comparing the results of 3 groups clinically there were remarkable change in gingival status in group IIb and IIa as compared to group I. this is due to the augmenting effect of $0.5 \%$ and $1 \%$ of curcumin with standard mechanical debridement of scaling and root planning. The antiinflammatory effect of curcumin could also be due to the hydroxyl and phenol groups in the molecule being essential for the inhibition of prostaglandin and leukotrienes. It is evident that on application of oral curcuma solution $(0.5$ and $1 \%)$ thrice daily for a episode of four weeks shows a tremendous reduce in inflammation and improve colour, contour of the gingiva, which in future will influence the treatment by the fading the bleeding on probing leading to easy handling of tissues, thus enabling us to arrest any further disease progression. The clinical effectiveness of curcumin concentrations over a longer period of time requires further studies and also there is scope for its comparison with other anti-inflammatory agents in management of periodontal diseases.

\section{CONCLUSION}

Although, SRP reduces inflammation but with curcumin application, reduction in inflammation was enhanced as observed in terms of reduction in gingival index and Bleeding on probing. The results highlights the fact that curcumin do have an additive role in reducing gingival bleeding (cardinal sign of gingivitis) supporting its anti-inflammatory and antioxidant role. It can be attributed to its antibacterial activity in addition which could help in decreasing the microbial load from the sulcus when used topically and as a gingival sulcularirrigant. Thus, the findings of the present study suggestthat curcumin, no doubt is a potent anti-inflammatory, antibacterial medicinal plant, and can be effectively used as an adjunct to mechanical debridement.

\section{ACKNOWLEDGEMENTS}

We are thankful of NBRI (National Botanical Research Institute) for providing curcumin extract and also thanks to our participants (patients) who showed compliance that helped the completion of this study.

\section{REFERENCES}

[1] Ashiq. S., Hussain. M., Ahmad. B., "Natural occurrence of mycotoxins in medicinal plants: a review," Fungal Genet Biol, vol 66: pp.1-10, 2014.

[2] Anirban. Chatterjee., Koel Debnath., and Nagabhushan Koratagere Hanumanta Rao.,"A comparative evaluation of the efficacy of curcumin and chlorhexidine mouthrinses on clinical inflammatory parameters of gingivitis: A double-blinded randomized controlled clinical study,"J Indian Soc Periodontol,21(2): 132-137, 2017.

[3] Jian Anurekha, Gupta VB., "Chemistry and pharmacological profile of Guggul- A review," Indian Journal of Treditional Knowledge. Vol. 5 no. 4, pp.478-483. Oct 2006.

[4] Kumar A, Singh AK, Kaushik MS, Mishra SK, Raj P, Singh PK, Pandey KD. "Interaction of turmeric (Curcuma longa L.) with beneficial microbes: a review.” 3 Biotech, vol. 7 no. 6 pp. 357

[5] M. Nagasri, M. Madhulatha, S. V. V. S. Musalaiah, P. Aravind Kumar, C. H. Murali Krishna,1 and P. Mohan Kumar,"Efficacy of curcumin as an adjunct to scaling and root planing in chronic periodontitis patients: A clinical and microbiological study,"J Pharm BioalliedSci, 7(Suppl 2): S554-S558, 2015.

[6] Gingivitis and periodontitis: Overview. Last Update: June 18, 2014. 
[7] LalithaTanjoreArunachalam, Uma Sudhakar, JohneyVasanth, Sophia Khumukchum, and VarshaVardhiniSelvam,"Comparison of anti-plaque and anti-gingivitis effect of curcumin and chlorhexidine mouth rinse in the treatment of gingivitis: A clinical and biochemical study," $J$ Indian SocPeriodonto, 21(6): 478-483, 2017.

[8] Abhishek Kandwal., Ravindra Kumar Mamgain., and PratibhaMamgain., "Comparative evaluation of turmeric gel with $2 \%$ chlorhexidine gluconate gel for treatment of plaque induced gingivitis: A randomized controlled clinical trial," 36(2): 145-150, 2015.

[9] Fabio Firenzuoli and Luigi Gori., "Herbal Medicine Today: Clinical and Research Issues" Evid Based Complement Alternat Med. Vol. 4 no. 1, pp 37-40. Sep 2007.

[10] Shweta S. Hugar, SuvarnaPatil, RenukaMetgud, BasavrajNanjwade, and Shivayogi M. Hugar.,"Influence of application of chlorhexidine gel and curcumin gel as an adjunct to scaling and root planing: A interventional study,"J Nat SciBiol Med, 7(2): 149-154, 2016.

[11] Gera M., Sharma N., Ghosh M..et al., "Nanoformulations of curcumin: an emerging paradigm for improved remedial application” Oncotarget; 8(39):66680-66698, 2017.

[12] Nilofer H, farjana, S C Chandrasekaran, and Bagavad Gitaet al., "Effect of Oral Curcuma Gel in Gingivitis Management - A Pilot Study". Journal of Clinical and Diagnostic Research. Vol-8 no., 12 pp.ZC08-ZC10., 2014.

[13] S.M Patel, NagulapalliVenkata KC, Bhattacharyya P, Sethi G, Bishayee A. "Potential of neem (Azadirachtaindica L.) for prevention and treatment of oncologic diseases," Seminars on cancer biology, pp.100-115, 2016.

[14] Rajan I, Rabindran R, Jayasree PR, Kumar PR., "Antioxidant potential and oxidative DNA damage preventive activity of unexplored endemic species of Curcuma,"Indian JExpBiol, 52(2), 133-8, 2014.

[15] Fine PD.,"A clinical trial to compare the effect of two antiseptic mouthwashes on gingival inflammation,"J Hosp Infect, 189-93, 1985.

[16] SS Yadav, Singh MK, Singh PK, Kumar V . "Traditional knowledge to clinical trials: A review on therapeutic actions of Emblica officinalis," Biomedicine and pharmacotherapy. pp.1292-1302, 2017.

[17] Loe H, Silness J. Periodontal disease in Pregnancy."Acta Odontologica Scand", 21:533-51,1963.

[18] Ainamo J, Bay I. Problems and proposals for recording gingivitis and plaque.”Int Dent J. ",25(4):229$35,1975$.

[19] Arora. R.B., Kapoor. V., Basu. N., Jain. AP., "Anti-inflammatory studies on Curcuma longa (turmeric)," Ind. J. MedRes, vol. 59,1289-1295.

[20] Ak. T., Gülçin. I., "Antioxidant and radical scavenging properties of curcumin,"Chem Biol Interact, 174(1), pp 27-37, 2008.

[21] Gopinath H., Karthikeyan K., "Turmeric: A condiment, cosmetic and cure".Indian J DermatolVenereolLeprol, Vol. 84 no. 1 pp. 16-21, 2018.

[22] Anuradha. BR., Bai. YD., Sailaja. S., Sudhakar. J., Priyanka. M., Deepika. V., et al., "Evaluation of Anti-Inflammatory Effects of Curcumin Gel as an Adjunct to Scaling and Root Planing: A Clinical Study,"Journal of International Oral Health, vol.7, no.7, 90-93, 2015. 